RASĀYAN J. Chem.

Vol. 13 | No. 3 |1824-1828| July - September | 2020 ISSN: 0974-1496 | e-ISSN: 0976-0083 | CODEN: RJCABP

\title{
NOVEL ONE POT SYNTHESIS OF 3, 5-(SUBSTITUTED PHENYL)-6 -PHENYL-3, 3A, 5, TRIHYDRO-2H-PYRAZOLO[3, 4-D] THIAZOLES BY GREEN METHOD
}

\author{
Sonal S. Mundhada \\ Department of Chemistry, Brijlal Biyani Science College, Sant Gadgebaba Amravati University \\ Amravati, Maharashtra, 444602, India. \\ *E-mail: sonal.mundhada@gmail.com
}

\begin{abstract}
Organic reactions on solid phase support represent eligibility and convenient way of traditional synthetic processes. Aluminates used in surface chemistry play an important role in their performance as catalyst and catalyst supporter. The one-pot solid-phase synthesis of novel 3,5-(substituted phenyl)-6- phenyl-3,3a,5,-trihydro-2H-pyrazolo[3,4d]thiazoles(IIa-IIh) by condensation of 2-(substituted phenyl)-3-phenylthiazolidin-4-one(Ia-Ih) (0.01M) with hydrazinehydrate $(0.015 \mathrm{M})$ and aromatic aldehyde $(0.02 \mathrm{M})$ in presence of solid-phase catalyst alumina under microwave irradiation, then it was cooled at room temperature and poured into dilute hydrochloric acid, the product thus obtained was filtered, washed with water and recrystallized from ethanol to get the compounds (IIa-IIh).

Keywords: Aromatic Aldehyde, Hydrazine Hydrate, 1, 3-Thiazolidin-4-ones.
\end{abstract}

(C) RASĀYAN. All rights reserved

\section{INTRODUCTION}

To reduce the environmental threat there is a need for clean chemical processes including monitoring analysis, synthetic procedures, catalysts and reaction conditions. To achieve these goals, green chemistry is being developed that based on innovative and unconventional synthetic procedures including reactions carried out in water ${ }^{1-2}\left(\right.$ normal $^{3}$ and superheated under high pressure $\left.{ }^{4}\right)$, in supercritical fluids $s^{5-6}$, in ionic liquids ${ }^{7}$, in micro emulsions ${ }^{8}$, in solvent-free conditions ${ }^{9}$, by ultrasounds ${ }^{10}$ and microwaves ${ }^{11-12}$ Pyrazoline derivatives have been found to possess considerable biological activities, which stimulate research activity in this field. ${ }^{13-17}$ Their prominent effects are antimicrobial ${ }^{18}$, stimulation of central nervous system $^{19}$, immunosuppressive ${ }^{20}$, anti-inflammatory ${ }^{21}$, anti-fertility ${ }^{22}$, anti-implantation ${ }^{23}$, insecticida ${ }^{24}$, antiproteolytic ${ }^{25}$ activities. Pyrazolines are found to be useful as antioxidant composition in polymers ${ }^{26}$ and the treatment of cerebral edema. ${ }^{27}$

\section{EXPERIMENTAL}

\section{Material and Methods}

All chemicals used were of analytical grade. All the synthesized compounds have been characterized based on chemical properties, elemental and spectral analysis. The melting points were measured in an open glass capillary and are uncorrected.IR spectra in $\mathrm{KBr}$ were recorded on instrument Perkin Elmer Spectrum RX-IFTIR. ${ }^{1} \mathrm{H}-\mathrm{NMR}$ spectra were recorded on FT NMR Spectrometer model Advance-II (Bruker) It's ${ }^{1} \mathrm{H}$ frequency is $400 \mathrm{MHz} 13 \mathrm{C}$ the frequency is $100 \mathrm{MHz}\left(\mathrm{CDCl}_{3}\right.$ and DMSO-d6) using TMS as an internal standard. All reactions were monitored by TLC using silica gel $60-\mathrm{f}-254$ plates. All reactions were carried out in a scientific microwave oven (Scientific microwave system model RG31lL1, $700 \mathrm{w}, 2450 \mathrm{MHz})$. Satisfactory C, H, N analysis were carried out for most of the compounds on Thermo Scientific (FLASH 2000) CHN Elemental Analyzer at RSIC, Punjab University, Chandigarh.

Synthesis of 3,5-(substituted phenyl)-6 phenyl-3,3a,5,-trihydro -2H-pyrazolo[3,4-d] thiazoles (IIaIIh)

The reaction is completed in the following two steps:

Rasayan J. Chem., 13(3), 1824-1828(2020)

http://dx.doi.org/10.31788/ RJC.2020.1335713 
RASĀYAN J. Chem.

Vol. 13 | No. 3 |1824-1828| July - September | 2020

Synthesis of 2-(substituted phenyl)-3-phenylthiazolidin-4-ones (Ia-Ih)

A neat reaction technology for one-pot synthesis of the starting material of 2-(substituted phenyl)-3phenylthiazolidin)-4-ones by condensation of different aromatic aldehydes $(0.01 \mathrm{M})$, aniline $(0.01 \mathrm{M})$, and thioglycolic acid $(0.01 \mathrm{M})$, Carried out under scientific microwave oven. The irradiation time is $1-1.5 \mathrm{~min}$. The reaction mixtures were cooled at room temperature and poured into ice-cold water. The products thus separated were filtered and crystallized from ethanol to get fine crystals of 2-(substituted phenyl)-3phenylthiazolidin-4-one. (Ia- Ih) compounds.

Synthesis of 3,5-(substitutedphenyl)-6 phenyl-3,3a,5,-trihydro -2H-pyrazolo[3,4-d] thiazole(IIa-IIh) This section reported the one-pot solid-phase synthesis of novel 3,5-(substitutedphenyl)-6 phenyl-3,3a,5,trihydro-2H-pyrazolo[3,4-d] thiazoles (IIa-IIh) by condensation of 2-(substituted phenyl)-3phenylthiazolidin-4-one( Ia- Ih) $(0.01 \mathrm{M})$ with hydrazine hydrate $(0.015)$ and aromatic aldehyde $(0.02 \mathrm{M})$ in presence of solid-phase catalyst Alumina under microwave irradiation, then mixture was cooled at room temperature and poured into dilute hydrochloric acid, the product thus obtained were filtered, washed with water and recrystallized from ethanol to get the compounds (IIa-IIh).

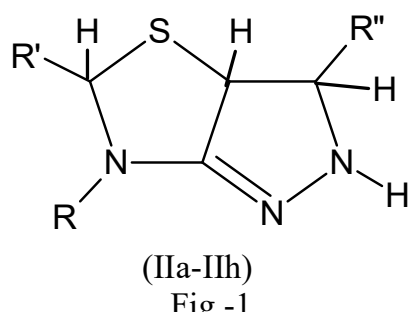

\section{Analytical Discussion}

Fig.-1

Synthesis of 3-(2,4-dicholorophenyl)-5,6-diphenyl-3,3a,5,-trihydro-2H-pyrazolo[3,4-d] thiazole(IIb)The compound ( $\mathrm{IIb}$ ) is a brown crystalline solid having M. P..254 ${ }^{\circ} \mathrm{C}$, molecular formula $\mathrm{C}_{22} \mathrm{H}_{17} \mathrm{Cl}_{2} \mathrm{~N}_{3} \mathrm{~S}$, $\% \mathrm{C}-61.97, \% \mathrm{~N}-9.86, \% \mathrm{~S}-7.52, \% \mathrm{H}-4.02 \% \mathrm{Cl}-16.63$.

\section{Spectral Study (IR Study in $\mathbf{c m}^{-1}$ )}

3332,m,NH Strech ,3032,s, C-H(aromatic), 2924,w, C-H stretch(aliphatic), 1952-1814,m, Combination band, $1671, \mathrm{~s}, \mathrm{C}=\mathrm{N}, 1595,1496,1456, \mathrm{~s}, \mathrm{C}=\mathrm{C}, 1496, \mathrm{~m}, \mathrm{NH}$ bend,1385,s,C-N, 1112,1075,s,C-Cl aryl, $898, \mathrm{~s}, 1,2,4$ trisubstituted oop, 730,693,s, Monosustituted oop.

(PMR in $\delta \mathrm{ppm})-3.54, \mathrm{~s}(\mathrm{NH}), 4.02, \mathrm{~d}(-\mathrm{CH}(\mathrm{J}=1.4)), 4.06, \mathrm{~d}(-\mathrm{CH}(\mathrm{J}=1.4), 6.5, \mathrm{~s},(-\mathrm{CH}), 7.40, \mathrm{~d}, 2 \mathrm{H},(\mathrm{Ar}-\mathrm{H}(-$ $\mathrm{CH}) \mathrm{J}=8.5 \mathrm{~Hz}, 7.31, \mathrm{~d}, 2 \mathrm{H},(\mathrm{Ar}-\mathrm{H}(-\mathrm{CH}) \mathrm{J}=8.5 \mathrm{~Hz}, 7.21-7.32, \mathrm{~m}, 8 \mathrm{H}, \mathrm{Ar}-\mathrm{H}$.

The CMR spectrum of compound (IIb) was reported in (DMSO-d6) with TMS as an internal standard. The observed chemical shifts can be correlated as follows: $\mathrm{C}_{3}=\mathrm{C}_{5}(63.48), \mathrm{C}_{3 \mathrm{a}}(32.73), \mathrm{C}_{6 \mathrm{a}}(170.49)$, $\mathrm{C}_{7}(140.04), \mathrm{C}_{8}=\mathrm{C}_{12}(125.48), \mathrm{C}_{9}=\mathrm{C}_{11}=\mathrm{C}_{17}=\mathrm{C}_{15}(128.62), \mathrm{C}_{10}(124), \mathrm{C}_{13}(136), \mathrm{C}_{14}=\mathrm{C}_{18}(129.97), \mathrm{C}_{16}(126.97)$, $\mathrm{C}_{19}(137.64), \mathrm{C}_{20}(128.42), \mathrm{C}_{21}=\mathrm{C}_{23}(128.67), \mathrm{C}_{22}=\mathrm{C}_{24}(126.36)$.

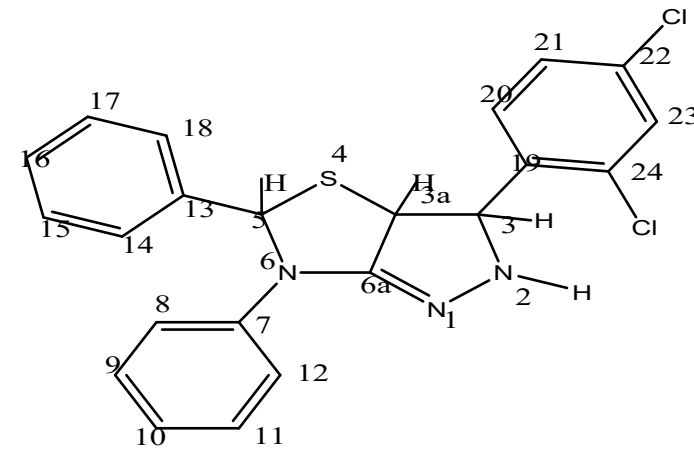

Fig.-2

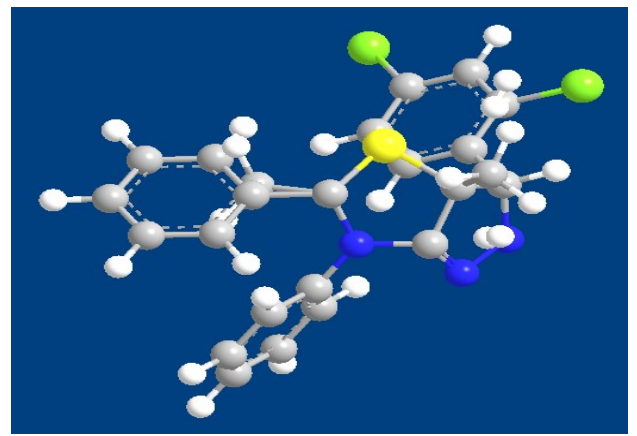

1825 
RASĀYAN J. Chem.

Vol. 13 | No. 3 |1824-1828| July - September | 2020

\section{Probable Mechanism}

In the present work, three-component condensation between thiazolidione, aldehyde and hydrazine hydrate over basic alumina in solvent free condition under microwave irradiation lead to the target molecules. In this proposed mechanism, the first step concerned with Knoevenagel condensation of thiazolidione and aldehyde in situ by absorption at basic alumina. Being M.W. transparent basic alumina provides surface and also catalyses the reaction $\beta$ unsaturated ketone through carbonium ion intermediate. In second step nucleophilic attack of nitrogen of the reagent on electron-deficient carbonyl carbon of thiazolidione and the carbonyl oxygen gets hydroxylated. At end nitrogen of the hydrazine hydrate bond with $\beta$ carbon of intermediate II leads to pyrazoline molecule through cyclodehydration.

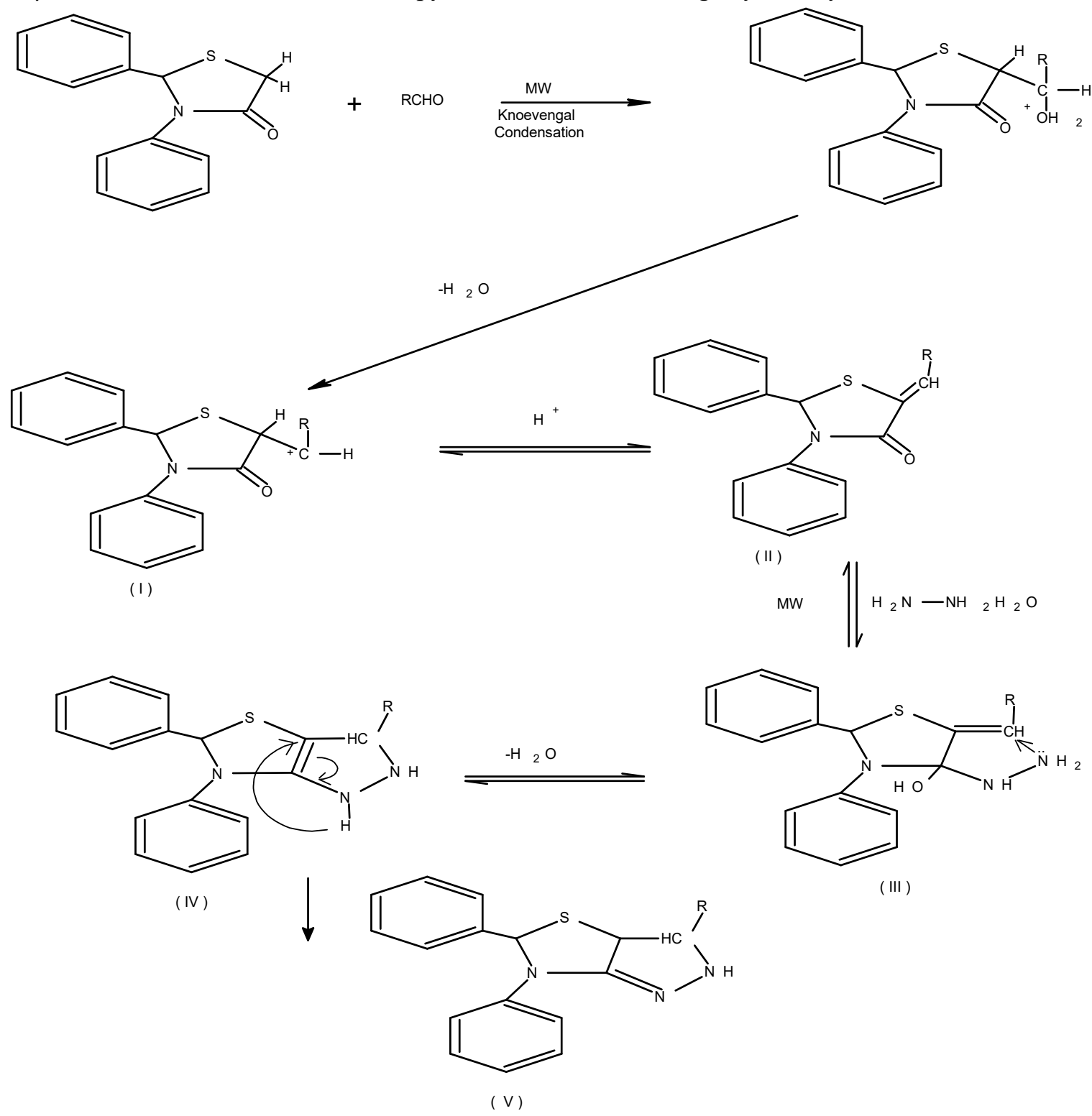

Scheme-1

Synthesis of 3-(4-bromophenyl)-5-(2,4-dicholorophenyl)-6-phenyl-3,3a,5,-trihydro-2H-pyrazolo[3,4d]thiazole (IId)

The compound (IId) is a brown crystalline solid having M. P..267 ${ }^{\circ} \mathrm{C}$, the molecular formula of the compound as $\mathrm{C}_{22} \mathrm{H}_{16} \mathrm{BrCl}_{2} \mathrm{~N}_{3} \mathrm{~S}$. 
RASĀYAN $J$. Chem.

Vol. 13 | No. 3 |1824-1828| July - September | 2020

Table-1: Synthesis of 3,5-(substituted phenyl)-6 phenyl-3,3a,5,-trihydro -2H-pyrazolo[3,4-d] thiazoles (IIa-IIh).

\begin{tabular}{|c|c|c|c|c|c|c|}
\hline S.No. & $\mathrm{R}$ & $\mathrm{R}^{\prime}$ & R" & M.P. $\left({ }^{0} \mathrm{C}\right)$ & M.F. & Yeild $(\%)$ \\
\hline IIa & $-\mathrm{C}_{6} \mathrm{H}_{5}$ & $-\mathrm{C}_{6} \mathrm{H}_{4} \mathrm{OH}$ & $-\mathrm{C}_{6} \mathrm{H}_{4} \mathrm{Br}$ & 231 & $\mathrm{C}_{22} \mathrm{H}_{18} \mathrm{BrN}_{3} \mathrm{OS}$ & 78 \\
\hline $\mathrm{IIb}$ & $-\mathrm{C}_{6} \mathrm{H}_{5}$ & $-\mathrm{C}_{6} \mathrm{H}_{5}$ & $-\mathrm{C}_{6} \mathrm{H}_{3} \mathrm{Cl}_{2}$ & 254 & $\mathrm{C}_{22} \mathrm{H}_{17} \mathrm{Cl}_{2} \mathrm{~N}_{3} \mathrm{~S}$ & 82 \\
\hline IIc & $-\mathrm{C}_{6} \mathrm{H}_{5}$ & $\mathrm{C}_{2} \mathrm{H}_{5}$ & $-\mathrm{C}_{6} \mathrm{H}_{4} \mathrm{Br}$ & 231 & $\mathrm{C}_{18} \mathrm{H}_{18} \mathrm{BrN}_{3} \mathrm{~S}$ & 84 \\
\hline IId & $-\mathrm{C}_{6} \mathrm{H}_{5}$ & $-\mathrm{C}_{6} \mathrm{H}_{3} \mathrm{Cl}_{2}$ & $-\mathrm{C}_{6} \mathrm{H}_{4} \mathrm{Br}$ & 267 & $\mathrm{C}_{22} \mathrm{H}_{16} \mathrm{BrCl}_{2} \mathrm{~N}_{3} \mathrm{~S}$ & 79 \\
\hline IIe & $-\mathrm{C}_{6} \mathrm{H}_{5}$ & $\begin{array}{c}-\mathrm{C}_{6} \mathrm{H}_{4}- \\
\mathrm{OCH}_{3}\end{array}$ & $-\mathrm{C}_{6} \mathrm{H}_{4} \mathrm{OH}$ & 284 & $\mathrm{C}_{23} \mathrm{H}_{21} \mathrm{~N}_{3} \mathrm{O}_{2} \mathrm{~S}$ & 85 \\
\hline IIf & $-\mathrm{C}_{6} \mathrm{H}_{5}$ & $-\mathrm{C}_{6} \mathrm{H}_{3} \mathrm{Cl}_{2}$ & $-\mathrm{C}_{6} \mathrm{H}_{4} \mathrm{Cl}$ & 251 & $\mathrm{C}_{22} \mathrm{H}_{16} \mathrm{Cl}_{3} \mathrm{~N}_{3} \mathrm{~S}$ & 87 \\
\hline IIg & $-\mathrm{C}_{6} \mathrm{H}_{5}$ & $\begin{array}{l}\mathrm{C}_{6} \mathrm{H}_{4-} \\
\mathrm{OCH}_{3}\end{array}$ & $-\mathrm{C}_{6} \mathrm{H}_{4} \mathrm{Br}$ & 258 & $\mathrm{C}_{23} \mathrm{H}_{20} \mathrm{BrN}_{3} \mathrm{OS}$ & 83 \\
\hline IIh & $-\mathrm{C}_{6} \mathrm{H}_{5}$ & $-\mathrm{C}_{6} \mathrm{H}_{4} \mathrm{OH}$ & $-\mathrm{C}_{6} \mathrm{H}_{4} \mathrm{Br}$ & 263 & $\mathrm{C}_{2} 2 \mathrm{H}_{18} \mathrm{BrN}_{3} \mathrm{OS}$ & 84 \\
\hline
\end{tabular}

\section{Spectral Study (IR in $\mathbf{C m}^{-1}$ )}

3362,m,NH; 3041,s, C-H (aromatic); 2977, w, C-H stretch(aliphatic); 1913,m, Combination band;1692,s, $\mathrm{C}=\mathrm{N} ; 1597,1490$ (s) $\mathrm{C}=\mathrm{C} ; 1410$ (s) $\mathrm{CH}_{2}$ bend; 1365(s) C-N , 1100,1071(s) C-Cl(aryl halide),1028,1010(s) C-Cl (aryl halide); 904(s) 1,2,4 trisubstituted oop.; 840(s) Parasubstituted oop.

(PMR in $\delta$ ppm ) 3.5(broad),-NH , 3.99(d) -CH(J=15.7), 4.0(d), -CH (J=15.7), 6.5(s)-CH , 7.47(d),2H Ar-H(-CH)J=8.46 Hz., 7.36(d), 2H Ar-H (-CH) J=8.46 Hz, 7.1-7.4(m) 8H Ar-H.

The CMR spectrum of compound (IId) was reported in (DMSO-d6) with TMS as an internal standard. There is good agreement in calculated and observed chemical shifts can be correlated as follows: $\mathrm{C}_{3}=\mathrm{C}_{5}$ (62.76), $\quad \mathrm{C}_{3 \mathrm{a}}(32.71), \mathrm{C}_{6 \mathrm{a}}(170.39), \mathrm{C} 7(139.56), \quad \mathrm{C}_{8}=\mathrm{C}_{12}(125.48), \quad \mathrm{C}_{9}=\mathrm{C}_{11}(129.24)$, $\mathrm{C}_{10}(119), \mathrm{C} 13(139.56), \mathrm{C}_{14}(131.37), \mathrm{C}_{15}=\mathrm{C}_{17}(128.67), \mathrm{C}_{16}=\mathrm{C}_{18}(139.43), \mathrm{C}_{19}(126.49), \mathrm{C}_{20}=\mathrm{C}_{24}(128.76)$, $\mathrm{C}_{21}=\mathrm{C}_{23}(131.57), \mathrm{C}_{22}(121.38)$.

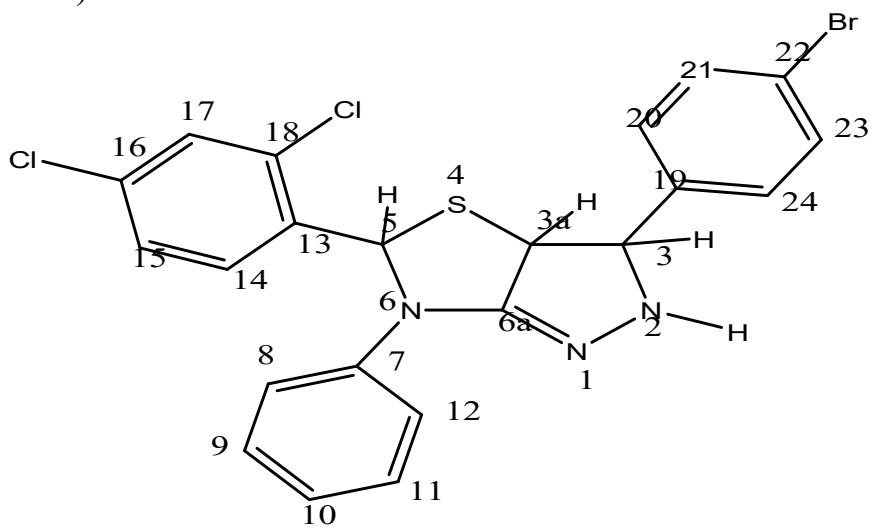

( IId)

Fig.-3

\section{RESULTS AND DISCUSSION}

From the chemical properties, elemental result and spectral data, the compounds 3, 5-(Substituted Phenyl)-6- Phenyl-3,3a, 5, Trihydro-2H-Pyrazolo [3, 4-D]Thiazoles ( IIa-IIh) show the confirmatory results.

\section{CONCLUSION}

The microwave technique has a beneficial effect on reaction if a mode reaction needs heating. Many new compounds 3, 5-(Substituted Phenyl)-6- Phenyl-3,3a, 5, Trihydro-2H-Pyrazolo [3, 4-D] Thiazoles ( IIaIIh) were successfully synthesized by microwave irradiation with high yield and short reaction time. Chemistry plays an integral part in our lives. Sustainability, eco-friendly and green chemistry are new principles that are guiding the development of the next generation of products and processes "Green chemistry is considered an essential piece of a comprehensive program to protect human health and the 
RASĀYAN J. Chem.

Vol. 13 | No. 3 |1824-1828| July - September | 2020

environment." In its essence green chemistry is a science-based non-regulatory and economically driven approach to achieving the goals of environmental protection and sustainable development.

\section{REFERENCES}

1. F. Fringuelli, O. Piermatti, F. Pizzo, L. Vaccaro, European Journal of Organic Chemistry 439,42(2001), DOI:10.1002/1099-0690(200102)2001:3<439::AID-EJOC439>3.0.CO;2-B

2. A. R. Katritzky, D. A. Nichols, M. Siskin, R. Murgan, M. Belasubramanian, Chemical. Reviews, 837, 101(2001), DOI: 10.1021/acs.energyfuels.9b01697

3. M. Siskin, A. R. Katritzky, Chemical. Reviews, 825, 101(2001), DOI:10.1021/ef00023a026

4. F. G. Klarner, V. Breitkopf, European Journal of Organic Chemistry, 57, 27(1999), DOI: 10.1021/acs.organomet.6b00886

5. A. Bailker et.al. Chemical Reviews, 99, 453(1999)

6. P. G. Jessop, T. Ikariya, R. Noyori, Chemical Reviews, 475, 99, (1999), DOI: 10.1021/acs.chemrev.7b00571

7. M. J. Earle, P. B. Mccormac, K. R. Seddon, Green Chemistry, 23, 1(1999), DOI:10.1039/A808052F

8. H. Krister, Advanced Colloid Interface Science, 131, 15, (1994), DOI:10.1016/00018686(94)80035-9

9. K. Tanaka, F. Toda, Chemical Reviews, 1025, 100(2000), DOI:10.1021/cr940089p

10. A. Fini, A. Breccia, Pure Applied Chemistry, 71, 573(1999), DOI:10.1351/pac199971040573

11. R. S. Verma, Green Chemistry, 1, 43(1999), DOI:10.1039/A808223E

12. L. Perreux, A. Loupy, Tetrahedron, 57, 9199(2001), DOI:10.1016/S0040-4020(01)00905-X Chemat $\mathrm{F}$

13. H.C. Garg and Chandraprakash, Journal of Pharmaceuticals Science, 14, 649(1971)

14. R.H. Ed. Pyrazoles, Pyrazolines, Pyrazolidines, Indazoles and Condensed Rings, Wily New York, Vol. 22, p. 180(1967)

15. J. Elguero, In. Comprehensive Heterocyclic Chemistry II, A.R. Katritzky, Scriven, Eds., Pergamon Press: Oxford; Vol. 3, p.1 (1996)

16. A. Levai, Khim, Journal of Heterocyclic Chemistry, 43(1), 111(1997), DOI:10.1002/jhet.5570430117

17. A. Levai, Journal of Heterocyclic Chemistry, 39(1), 1 (2002), DOI:10.1002/jhet.5570390101

18. K. Ramalingham, G.X. Thyvekikakath, N.N. Durham, A.E. Ealick, D. Vander Helm, Journal of Medicinal Chemistry, 20, 847(1977)

19. R.E. Brown, Jr. Shavrel, J.US Patent, 3, 624 (1972) and Chemistry Abstract, 76, 59618 (1972)

20. J.G. Lombardino, I.G. Otterness, Journal of Medicinal Chemistry, 24, 830, (1981)

21. J.P. Dusza, J.P. Joseph and S. Burnstan, U.S. USA360 680 (CI 54862 071).

22. R.N. Iyer and R. Gopalchand, Indian Journal of. Chemistry, 15 B, 194(1976).

23. S. Kumar and N. Rastogi, Indian Journal of Chemistry, 26B, 968(1987)

24. R. Von Hes, and A.C. Crossurt, European Patent, EP 65, 334 (CI CO7D 231/06) (1982), Chemistry Abstract, 598, 107287n ,(1983)

25. K. Raman, B.R. Pandey, J.P. Barthwal and S.S. Parmar, European journal of Medicinal Chemistry. Chemical Theory, 15, 567(1980) and Chemical Abstract, 94, 95909f(1981)

26. M. Morigaki and N. Seto, Japan (Kokai), Chemical Abstract, 63, 115, 866(1989)

27. H. Yamashita, K. Okumura, H. Lizuka and N. Ohto, European Patent(1990)

[RJC-5713/2019] 